Ciência e Natura, Santa Maria, v. 37 Ed. Especial PROFMAT, 2015, p.203-213

Revista do Centro de Ciências Naturais e Exatas - UFSM

ISSN impressa: 0100-8307 ISSN on-line: 2179-460X

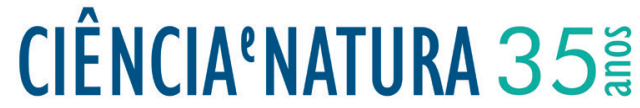

\section{O Teorema de Pick}

\author{
The Pick's Theorem
}

\section{Joelson Dayvison Veloso Hermes*1}

${ }^{1}$ Instituto Federal de Educação, Ciência e Tecnologia do Sul de Minas Gerais. MG, Brasil

\begin{abstract}
Resumo
Neste trabalho o principal tema abordado éo Teorema de Pick. Esse teorema se refere ao cálculo de áreas de polígonos com vértices nos pontos de uma rede no plano, tal cálculo se resume em uma simples relação entre o número de pontos da rede, localizados no interior do polígono e o número de pontos da fronteira do polígono que pertencem à rede. Procura, ainda, antes de apresentar sua demonstração, alinhar as ideias centrais de sua demonstração, para que se tenha argumentos suficientes para tal. Propomo-nos estabelecer a relação entre este método e o já conhecido Teorema de Euler, além de apresentarmos uma aplicação aritmética para esse teorema. A atenção voltada para esse teorema deve-se à sua aparente simplicidade e ao fato de acreditamos que ele pode despertar o interesse de alguns estudantes pelo assunto.
\end{abstract}

Palavras-chave: Teorema de Pick. Cálculo de áreas. Rede de pontos

\begin{abstract}
This work has as a main theme the Pick's Theorem. This theorem refers to the calculation of area of polygons with vertices at points of a flat network, such a calculation can be summarized in a simple relation between the number of points of a network, located in the interior of a polygon and the number of points of the border of the polygon that belongs to the network. Research, before, presenting your demonstration, align the central ideas of your demonstration, so that there are sufficient arguments to support it. We propose to establish a relation between this method and the well known theorem of Euler, besides presenting an arithmetical application for this theorem. The attention given to this theorem is well deserved due to its apparent simplicity and for the fact that it can awaken the interest of some students for the subject.
\end{abstract}

Keywords: The Pick's Theorem. Calculation of area. Network of points 


\section{Introdução}

O conceito de área está presente em diferentes momentos da educação, da Educação Infantil ao Ensino Médio, e até mesmo no Ensino Superior. Na maioria das vezes, o cálculo de área é feito através da decomposição ou composição em figuras de áreas conhecidas, ou ainda por meio de estimativas, o que nem sempre é tarefa fácil.

Assim, pretende-se apresentar um novo método para o cálculo de área, a partir da contagem de pontos, o que é de certa forma surpreendente, pois o processo até então usado envolve medições de grandezas contínuas. Esse será substituído por uma contagem de grandezas discretas, ou seja, um novo método, o fascinante Teorema de Pick.

Neste trabalho buscaremos mostrar um raciocínio que leva de modo intuitivo a sua fórmula e também as hipóteses e condições para sua aplicação.

\section{Um pouco de História}

Georg Alexander Pick nasceu em uma família judia no ano de 1859 em Viena. Sua mãe era Josefa Schleisinger e seu pai foi Adolf Josef Pick, diretor de uma instituição privada. Georg foi educado em casa por seu pai até os onze anos de idade, depois ele entrou na quarta classe do Leopoldstaedter Communal Gymsasium, ficando nesta escola até 1875, quando realizou exames de qualificação para universidade. Ele entrou na Universidade de Viena em 1875. Ele publicou seu primeiro artigo matemático, no ano seguinte, com apenas dezessete anos de idade. Estudou matemática e física, graduando-se em 1879 com uma qualificação que lhe permitiria ensinar ambas as disciplinas.

Seu trabalho foi extremamente amplo no campo da matemática, em sua gama de 67 artigos foram abordados muitos tópicos, tais como Álgebra Linear, Análise Funcional, Cálculos de Integrais e Geometria. No entanto mais da metade de seus artigos estavam em funções de uma variável complexa, equações diferenciais e geometria diferencial. Termos como Matrizes Pick, Interpolação PickNevanlinna e o Lema Schwarz-Pick são usados até hoje. $\mathrm{O}$ seu artigo mais lembrado, no entanto, é o Teorema de Pick - Pick's Theorem - que apareceu no seu artigo de oito páginas Geometrisches zur Zahlenlehre publicado em Praga em 1899. O resultado de seu trabalho não recebeu muita atenção inicialmente. Todavia, após a sua citação em 1969 pelo matemático H. Steinhaus, que o incluiu em um de seus livros, este resultado atraiu muita atenção e admiração por ser simples e elegante.

Pick tinha sido eleito como membro da Academia das Ciências e das Artes da República Tcheca, mas após os nazistas assumirem, ele foi excluído da Academia. Pick foi enviado para Theresienstadt em 13 de Julho de 1942, morrendo duas semanas mais tarde aos 82 anos.

\section{O Teorema de Pick}

Antes de enunciarmos o Teorema de Pick, devemos definir precisamente o conceito de rede de pontos, pois tal teorema é definido para esse contexto.

Uma rede no plano é um conjunto infinito de pontos dispostos regularmente ao longo de retas horizontais e verticais, de modo que a distância de cada um deles aos pontos mais próximos na horizontal ou na vertical é igual a 1. Tomando um sistema de coordenadas cartesianas, com origem num ponto da rede, um eixo na direção horizontal e outro na vertical, a rede pode ser descrita como o conjunto de todos os pontos do plano cujas coordenadas $(m, n)$ são números inteiros. Ver [1]. Um polígono simples é um polígono cuja fronteira é uma poligonal fechada que pode ser percorrida inteiramente sem passar duas vezes pelo mesmo vértice. Sendo assim enunciamos o Teorema de Pick.

Teorema 3.1 A área de um polígono simples cujos vértices são pontos de uma rede é dada pela fórmula

$$
A=\frac{f}{2}+I-1,
$$

onde fé o número de pontos da rede situados sobre o contorno do polígono e I é o número de pontos da rede situados no interior do polígono, que serão chamados aqui respectivamente de pontos da fronteira e pontos do interior. 
Vejamos um exemplo.

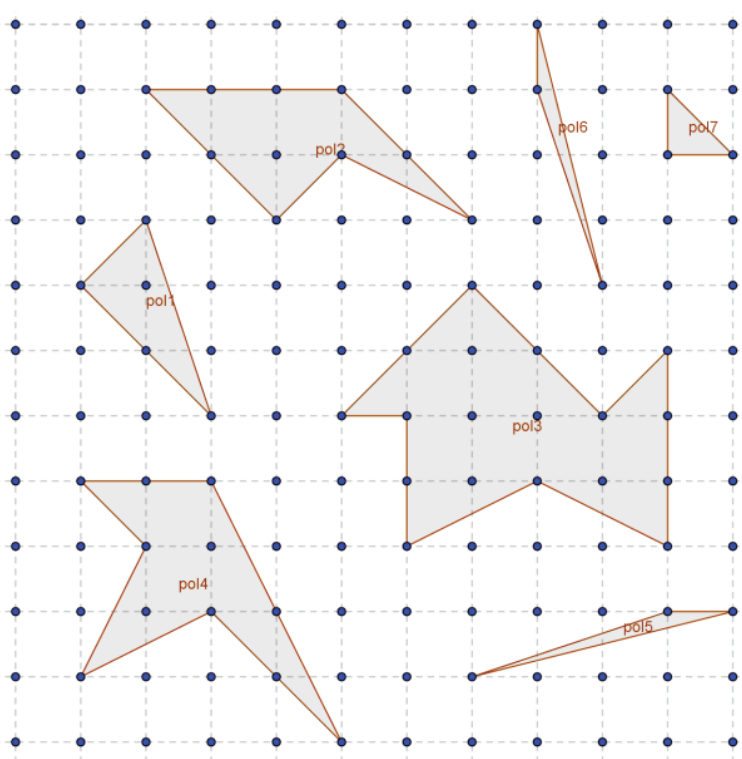

Figura 1

$$
\begin{gathered}
A(\text { pol } 1)=\frac{4}{2}+1-1=2 \\
A(\text { pol } 2)=\frac{9}{2}+1-1=4,5 \\
A(\text { pol } 3)=\frac{13}{2}+5-1=10,5 \\
A(\text { pol } 4)=\frac{9}{2}+2-1=5,5 \\
A(\text { pol } 5)=\frac{3}{2}+0-1=0,5 \\
A(\text { pol6 })=\frac{3}{2}+0-1=0,5 \\
A(\text { pol } 7)=\frac{3}{2}+0-1=0,5
\end{gathered}
$$

Atualmente, pode se encontrar facilmente uma série de demonstrações desse teorema, a escolha da demonstração a qual iremos descrever se deve a evidente relação com os objetivos propostos pelo trabalho, ou seja, ao desenvolvermos os argumentos necessários para tal demonstração veremos claramente a relação entre o Teorema de Pick e o Teorema de Euler para poliedros planos e uma aplicação aritmética do Teorema de Pick. Essa demonstração é uma síntese e adaptação da publicada no livro Meu professor de Matemática e outras histórias, ver [1], neste sentido, para atingir nosso objetivo, a seguir demonstraremos três lemas.

O primeiro lema é sobre a área de um Triângulo Fundamental. Um triângulo é dito Fundamental quando tem os três vértices e mais nenhum outro ponto, da fronteira ou do interior, sobre a rede.
Lema 3.1 A área de um triângulo fundamental é igual $a \frac{1}{2}$.

Demonstração: Considere $A(0,0)$ e $B(m, n)$ as coordenadas dos dois primeiros vértices do triângulo fundamental $A B C$. Analisando as coordenadas do ponto $B$, percebe-se que caso $d$ seja um divisor comum de $m$ e $n$ maior que 1 , o ponto $P(m / d, n / d)$ estaria na rede $\mathrm{e}$ consequentemente no interior do segmento $A B$, como se pode ver na Figura 2. Sendo assim o triângulo $A B C$ não seria fundamental, contrariando nossa hipótese. Logo $d$ deve ser igual a 1, ou seja, $m$ e $n$ são primos entre si, pois somente assim o triângulo $A B C$ será fundamental.

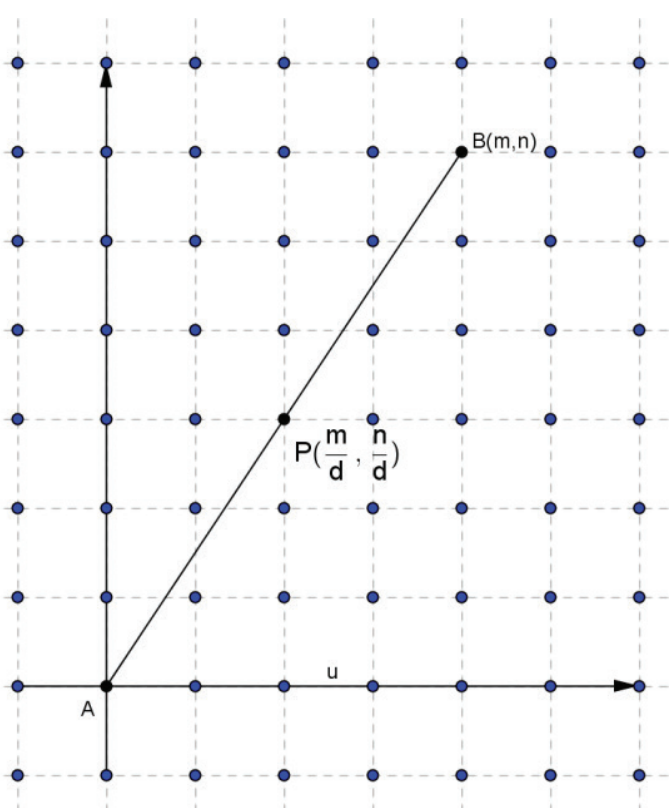

Figura 2

Analisando agora o ponto $C$, o terceiro vértice do triângulo $A B C$, e considerando $m \neq 0$, temos que a equação da reta $r$, que passa por $C$ e é paralela a $A B$, é $y=(n / m) x+b$, onde $b$ é a ordenada do ponto $D(0, b)$, ponto onde a reta $r$ corta o eixo vertical. Sendo assim se tomarmos qualquer triângulo com base $A B$ e com o seu terceiro vértice sobre a reta $r$, sua área será a mesma do triângulo $A B C$. Por conveniência vamos calcular a área do triângulo $A B D$ que possui base $|b|$ e altura $|m|$, como se pode ver na Figura 3, então a área do triângulo $A B D$ que designaremos por $|A B D|$ é igual a

$$
\frac{|b m|}{2}
$$

ou seja,

$$
|\mathrm{ABD}|=\frac{|b m|}{2} .
$$


Como

$$
|\mathrm{ABD}|=|\mathrm{ABC}|,|\mathrm{ABC}|=\frac{|b m|}{2} .
$$

Considerando agora uma reta $s$ qualquer, também paralela a $A B$, com equação $y=(n / m) x+$ $\beta$, como vimos anteriormente $\beta$ é a ordenada onde essa reta corta o eixo vertical. Tomando um ponto $E(s, t)$ arbitrário e pertencente a reta $s$, temos:

$$
\begin{aligned}
& t=\frac{n}{m} s+\beta, \\
& \beta=t-\frac{n}{m} s, \\
& \beta=\frac{m t-n s}{m} .
\end{aligned}
$$

Sabemos ainda que dentre todas as retas paralelas a $A B$ e que passa por algum ponto da rede, a mais próxima de $A B$ é a reta $r$, a qual passa pelo ponto $C$, onde teremos $\beta=b$. Observando a Figura 3 percebemos que o $|b|$ é o menor valor positivo que $|\beta|$ pode assumir.

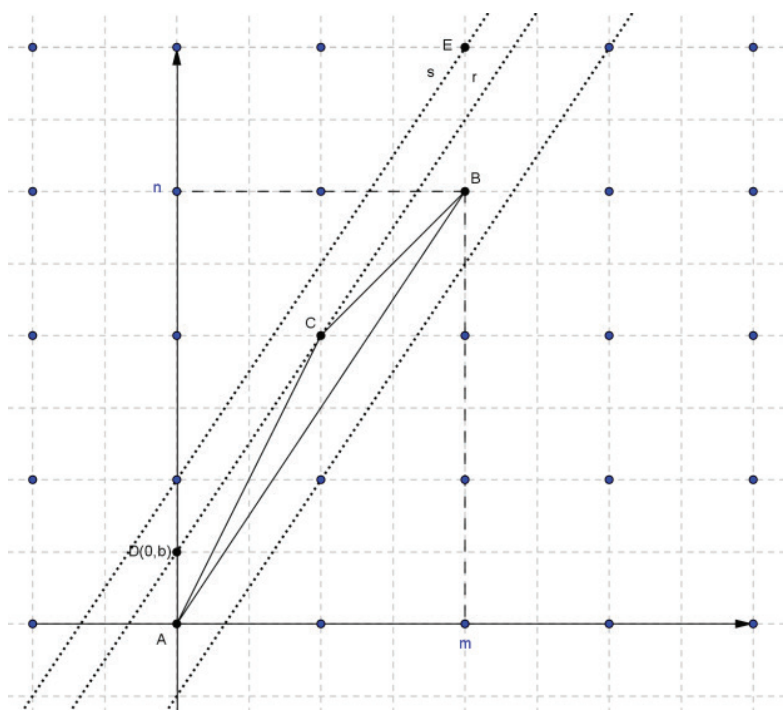

Figura 3

É bem conhecido que dois números inteiros $m$ e $n$ são primos entre si se, e somente se, existem números inteiros $s$ e $t$ tais que $t m-s n=1, \log$ o o menor valor positivo que $|\beta|$ pode assumir é $\frac{1}{m^{\prime}}$ $\log 0$

$$
|b|=\frac{1}{|m|}
$$

e como

$$
|A B C|=\frac{|b m|}{2}
$$

segue que

$$
|A B C|=\frac{1}{2} .
$$

Agora só nos resta mostrar o caso em que $m=$ 0 . Mas $m=0$ obriga $n= \pm 1$, com isso o triângulo $A B C$ passa a ser um triângulo retângulo cuja área é a metade de um quadrado unitário da rede, logo sua área também é $1 / 2$.

No segundo lema vamos fazer a Decomposição de um polígono e mostrar que qualquer polígono simples pode ser decomposto em um número finito de triângulos justaposto, pois assim a demonstração do teorema recai apenas na soma das áreas dos $n$ triângulos nos quais o polígono foi decomposto.

Lema 3.2 Todo polígono de $n$ lados pode ser decomposto como a reunião de $n-2$ triângulos justapostos, cujos vértices são do polígono dado.

Demonstração: Dividiremos a demonstração em dois casos, primeiramente analisaremos os polígonos convexos e posteriormente os não convexos.

Caso 1 - Polígonos convexos.

Seja $P=A_{1} A_{2} \ldots A_{n}$ um polígono convexo, escolhemos então um vértice $A_{i}$ desse polígono e traçamos as diagonais a partir dele. Assim o polígono $P$ ficará divido em $\quad(n-2)$ triângulos justapostos.

Caso 2 - Polígonos não convexos.

Admitindo que o teorema não seja verdadeiro, então existe um polígono $P \operatorname{com} n$ lados, o qual não pode ser decomposto em triângulos. Assumimos ainda que $n$ seja o menor número natural para o qual o polígono $P$ não pode ser decomposto. Tomamos agora uma reta $r$ que não intercepte $P$ e não seja paralela a nenhum de seus lados. Chamaremos de $B$ o vértice do polígono que esteja mais próximo de $r$, e de $A$ e $C$ os vértices adjacentes a $B$. Com isso teremos dois casos possíveis.

$1^{\circ}$ caso: $A, B$ e $C$ são os únicos vértices de $P$ que estão contidos na região triangular $A B C$. 


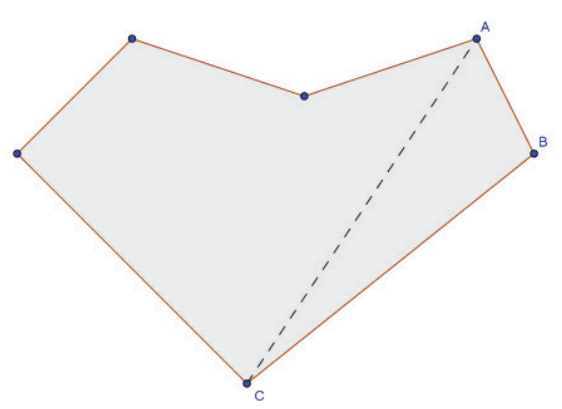

Figura 4

Quando ligamos os pontos $A$ e $C$ passamos a ter um novo polígono, o qual foi gerado a partir de $P$, substituindo os lados $A B$ e $B C$ pelo lado $A C$, a esse polígono daremos o nome de $P^{\prime}$. O novo polígono passa a ter agora $(n-1)$ lados, o que nos permite fazer sua decomposição, uma vez que assumimos anteriormente que $n$ seria o menor número para o qual o polígono não poderia ser decomposto. Assim que unimos $P^{\prime}$ ao triângulo $A B C$, obtemos a decomposição do polígono $P$, o que é uma contradição, já que assumimos inicialmente que um polígono com $n$ lados não poderia ser decomposto. Isso conclui a demonstração desse caso.

$2^{\text {o }}$ caso: A região triangular $A B C$ contém outros vértices do polígono $P$ Além de $A, B$ e $C$.

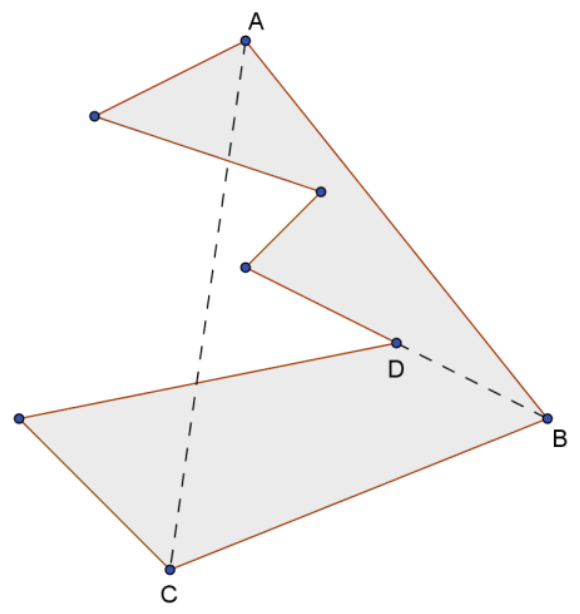

Figura 5

Para esse caso usaremos a seguinte estratégia: entre os pontos pertencentes à região triangular $A B C$ escolhemos o mais distante de $A C$, para que ao traçarmos a diagonal $B D$ esta não intercepte nenhum dos lados de $P$. Ao ligarmos os pontos $B$ e $D$, o polígono se divide em dois polígonos $P^{\prime}$ e $P^{\prime \prime}$. Mas tanto $P^{\prime}$ quanto $P^{\prime \prime}$ tem número de lados menores que do polígono $P$, então como assumimos que $n$ seria o menor número possível para o qual $P$ não poderia ser decomposto, conclui-se que $P^{\prime}$ e $P^{\prime \prime}$ podem ser decompostos. Unindo então $P^{\prime}$ e $P^{\prime \prime}$ encontramos a decomposição de $P$, o que é um absurdo, já que assumimos que $P$ não poderia ser decomposto. Isso completa a demonstração deste lema.

A demonstração dos lemas anteriores representa um grande avanço para conseguirmos demonstrar o teorema de Pick, mas para isso devemos dar mais um passo. Considere então o próximo lema como em [1].

\section{Demonstração do Teorema de Pick}

Agora sim temos argumentos suficientes para demonstrar o Teorema de Pick. Pois para isso precisamos contar o número $T$ de triângulos fundamentais em que o polígono pode ser decomposto, o que já vimos que é possível pelo Lema 3.3, e em seguida multiplicar esse número $T$ por sua área, que em virtude do Lema 3.1 sabemos que vale $1 / 2$.

Usaremos aqui o mesmo artifício usado para provar o Teorema de Euler para poliedros convexos, ou seja, vamos descobrir o número $T$ de triângulos fundamentais através da soma dos ângulos internos de todos os triângulos fundamentais que compõe o polígono.

Sabemos que a soma dos ângulos internos de um triângulo é igual a $\pi$, logo, a soma dos ângulos internos de todos os triângulos fundamentais que compõem o polígono é igual a $T \pi$. Outra forma de fazer essa contagem seria da a seguinte: Considere,

$S_{F}=$ soma dos ângulos com vértice na fronteira do polígono,

$S_{I}=$ soma dos ângulos com vértice no interior do polígono,

$S$ = soma dos ângulos internos dos $T$ triângulos fundamentais que compõe o polígono, $S=S_{F}+S_{I}$,

$f^{\prime}=$ número de vértices do polígono,

$f^{\prime \prime}=$ número de pontos sobre a fronteira do polígono, mas não vértices,

$f=$ pontos da fronteira do polígono, $f=f^{\prime}+f^{\prime \prime}$. 
Sendo assim temos que, $S_{f}=\left(f^{\prime}-2\right) \pi+f^{\prime \prime} \pi$, onde $\left(f^{\prime}-2\right)$ é o número de triângulo no qual o polígono foi decomposto, $\left(f^{\prime}-2\right) \pi$ é a soma dos ângulos interno do polígono e $f^{\prime \prime} \pi$ é a soma dos ângulos com vértices sobre a fronteira do polígono, mas que não são vértices, logo,

$$
\begin{aligned}
S_{f} & =\left(f^{\prime}-2\right) \pi+f^{\prime \prime} \pi \\
& =f^{\prime} \pi-2 \pi+f^{\prime \prime} \pi \\
& =\left(f^{\prime}-2+f^{\prime \prime}\right) \pi .
\end{aligned}
$$

Como $f=f^{\prime}+f^{\prime \prime}$ temos,

Temos também,

$$
S_{F}=(f-2) \pi .
$$

$$
S_{I}=I 2 \pi
$$

Logo,

$$
\begin{aligned}
S & =S_{F}+S_{I} \\
& =(f-2) \pi+I 2 \pi \\
& =f \pi-2 \pi+I 2 \pi \\
& =(f-2+2 I) \pi .
\end{aligned}
$$

Comparando o resultado das duas contagens temos,

$$
T \pi=(f-2+2 I) \pi,
$$

portanto

$$
T=f+2 I-2,
$$

que é o número de triângulos fundamentais no qual o polígono foi decomposto.

Logo, a área do polígono será $T$ vezes a área de um triângulo fundamental:

$$
\begin{aligned}
A(P) & =(f+2 I-2) \frac{1}{2}, \\
& =\frac{f}{2}+I-1,
\end{aligned}
$$

e o Teorema está provado.

\section{Uma extensão do Teorema de Pick}

Utilizando as ideias contidas em [4], vamos encontrar uma generalização do Teorema de
Pick que possa ser usada para calcular a área de uma região poligonal reticulada com buracos, ou seja, calcular a área de polígonos com buracos. Mas primeiramente devemos definir de forma mais precisa o que vem a ser uma região poligonal reticulada com buracos.

Definição 5.1 Uma região do plano delimitada por um polígono simples se chama uma região poligonal. Um buraco B de uma região poligonal P é uma região poligonal delimitada por um polígono simples contido em $P$.

Definição 5.2 Uma região poligonal $P$ tem $n$ buracos se existirem $n$ buracos, $B_{1}, \ldots, B_{n}$, em $P$ tais que para cada par $B_{i}, B_{j}$, com $i \neq j$, os polígonos que delimitam $B_{i}$ e $B_{j}$ são disjuntos e para todo índice $i$ o buraco $B_{i}$ não é um buraco de nenhum $B_{j}$, para todo $i \neq j$.

Definição 5.3 Uma região poligonal se dirá reticulada se os vértices do polígono se encontram nos pontos da rede, e uma região reticulada com buracos será uma em que os buracos estão delimitados por polígonos reticulados.

Teorema 5.1 Se R é uma região poligonal reticulada com $n$ buracos, então:

$$
A(R)=\frac{f}{2}+I-1+n,
$$

onde $f$ e I são, respectivamente, os números de pontos na fronteira e no interior de $R$.

Demonstração: Considerando uma região poligonal reticulada $R$ com os buracos $B_{1}, \ldots, B_{n}$, como representado na Figura 6.

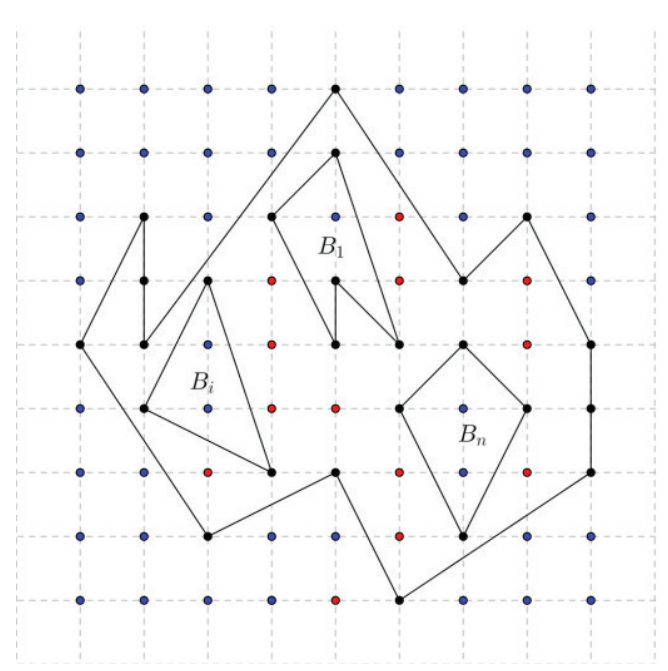

Figura 6 
Chamaremos de $P_{0}$ o polígono que delimita externamente a região poligonal $R$ e de $B_{i}, 1 \leq i \leq$ $n$, seus respectivos buracos. De acordo a definição dada anteriormente para uma região poligonal reticulada com buracos, vemos claramente que o Teorema de Pick se aplica tanto para $P_{0}$ como para os buracos.

Fazendo isso para $P_{0}$ e $B_{i}$ teremos respectivamente:

$$
A\left(P_{0}\right)=\frac{f_{0}}{2}+I_{0}-1 \text { e } A\left(B_{i}\right)=\frac{f_{i}}{2}+I_{i}-1
$$

Sendo assim a área de $R$ será $A(R)=A\left(P_{0}\right)-A\left(B_{i}\right)$, ou seja, $A(R)=A\left(P_{0}\right)-A\left(B_{1}\right)-A\left(B_{2}\right)-\ldots-A\left(B_{n}\right)$,

e aplicando o Teorema de Pick temos:

$$
\begin{gathered}
A(R)=\frac{f_{0}}{2}+I_{0}-1-\left(\frac{f_{1}}{2}+I_{1}-1\right)-\left(\frac{f_{2}}{2}+I_{2}-1\right)-\cdots-\left(\frac{f_{n}}{2}+I_{n}-1\right) \\
A(R)=\frac{f_{0}}{2}+I_{0}-1-\frac{f_{1}}{2}-I_{1}+1-\frac{f_{2}}{2}-I_{2}+1-\cdots-\frac{f_{n}}{2}-I_{n}+1 \\
A(R)=\frac{f_{0}}{2}-\frac{f_{1}}{2}-\frac{f_{2}}{2}-\cdots-\frac{f_{n}}{2}+I_{0}-I_{1}-I_{2}-\cdots-I_{n}-1+1+1+\cdots+1 \\
A(R)=\frac{f_{0}}{2}-\frac{f_{1}}{2}-\frac{f_{2}}{2}-\cdots-\frac{f_{n}}{2}+I_{0}-I_{1}-I_{2}-\cdots-I_{n}-1+n,
\end{gathered}
$$

Fazendo $I^{*}=I_{0}-I_{1}-I_{2}-\cdots-I_{n}$, temos:

$$
A(R)=\frac{f_{0}}{2}-\frac{f_{1}}{2}-\frac{f_{2}}{2}-\cdots-\frac{f_{n}}{2}+I^{*}-1+n,
$$

Agora usamos o artifício de somar e subtrair $f_{i}$ na última equação, obtemos,

$$
\begin{gathered}
A(R)=\frac{f_{0}}{2}-\frac{f_{1}}{2}+f_{1}-\frac{f_{2}}{2}+f_{2}-\cdots-\frac{f_{n}}{2}+f_{n}-f_{1}-f_{2}-\cdots-f_{n}+I^{*}-1+n \\
A(R)=\frac{f_{0}}{2}+\frac{f_{1}}{2}+\frac{f_{2}}{2}+\cdots+\frac{f_{n}}{2}-f_{1}-f_{2}-\cdots-f_{n}+I^{*}-1+n \\
A(R)=\frac{f_{0}+f_{1}+f_{2} \cdots+f_{n}}{2}+I^{*}-f_{1}-f_{2}-\cdots-f_{n}-1+n .
\end{gathered}
$$

Mas $f_{0}+f_{1}+f_{2} \ldots+f_{n}=f$, pois é a soma de todos os pontos pertencentes à fronteira de $R$ e $I^{*}-f_{1}-\cdots-f_{n}=I$, já que são todos os pontos internos de $P_{0}$ menos os internos e da fronteira dos buracos, logo o que resta são os pontos internos de $R$, com isso teremos:

$$
A(R)=\frac{f}{2}+I-1+n
$$

\section{Relação entre o Teorema de Pick e o Teorema de Euler para poliedros planos}

A demonstração do Teorema de Pick aqui apresentada nos sugere uma relação com o Teorema de Euler para poliedros planos, tendo em vista que essa demonstração imita argumentos usados para provar a fórmula de Euler.

Considere um polígono simples $P$ o qual foi decomposto em polígonos menores, que serão chamados de "faces", cada lado de uma dessas faces será chamado de "arestas", então, em analogia com o caso de poliedros convexos, as letras $F, A$ e $V$ representarão, respectivamente, o número de faces, de arestas e de vértices de $P$. 
O teorema de Euler nos diz que $F-A+V=1$. Mas é importante lembrar que tal relação só é válida caso sejam respeitadas as seguintes condições para a decomposição do polígono $P$ : duas faces quaisquer da decomposição, ou são disjuntas, ou têm um vértice em comum, ou têm uma ou mais arestas inteiras em comum. Um polígono $P$ munido de uma decomposição nessas condições é chamado de poliedro plano. A Figura 7 nos mostra um exemplo de um poliedro plano e ainda a relação de Euler para esse poliedro.

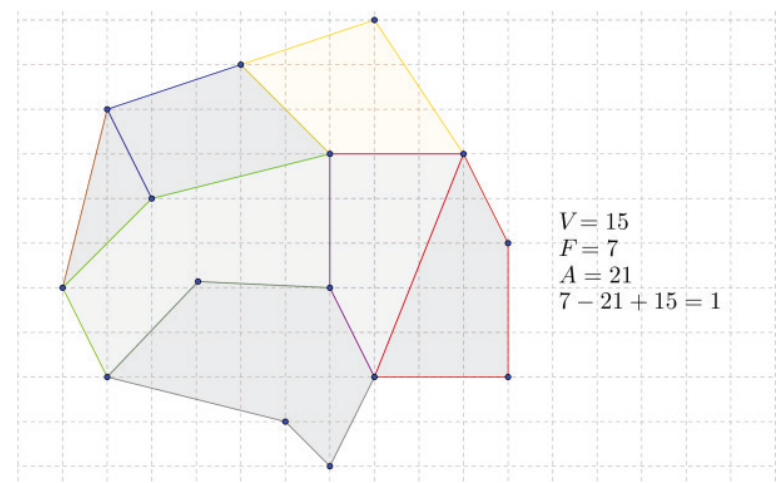

Figura 7

A relação entre o Teorema de Pick e o Teorema de Euler para poliedros planos é a seguinte.

Teorema 6.1 A fórmula de Euler para poliedros planos implica o Teorema de Pick.

Demonstração: Seja um poliedro plano $P$, se decompusermos cada uma de suas faces em triângulos, sem acrescentar novos vértices, percebe-se que os valores de $A$ e $F$ se modificarão, no entanto a relação $F-A+V=1$ se mantêm inalterada, pois quando se acrescenta uma aresta o número de faces também aumenta em uma unidade, com isso, esses incrementos se cancelam. Sendo assim não há perda de generalidade em considerar todas as faces como sendo triangulares. Mas será que essa relação se mantem caso o polígono seja decomposto em triângulos fundamentais, já que tal decomposição altera o número de vértice? A resposta para essa pergunta é sim. A demonstração desse fato se encontra no apêndice.

Analisando o número de arestas, percebemos que cada aresta externa $A_{e}$ é lado de apenas uma face e que cada aresta interna $A_{i}$ é lado de duas faces, com isso temos a seguinte relação,

$$
3 F=A_{e}+2 A_{i}
$$

Como a figura que limita o poliedro é um polígono simples, temos que o número de lados, que são as arestas exteriores, é igual ao número de vértices. Mas como cada face é um triângulo fundamental, ou seja, os únicos pontos do triângulo pertencentes à rede são seus vértices, então o número de vértices é igual ao número de pontos da fronteira $f$ do polígono, logo a equação anterior pode ser escrita da seguinte forma,

$$
3 F=2 A_{i}+f,
$$

temos também que o número de arestas interiores é igual ao número total $A$ de arestas, menos o número de arestas exteriores $f$, então,

$$
3 F=2(A-f)+f
$$

o que implica

$$
A=\frac{3 F+f}{2} .
$$

Com relação aos vértices, lembrando-se de que todas as faces são triângulos fundamentais, temos que o número de vértices $V$ é igual à soma dos pontos da fronteira $f$ com os pontos do interior $I$, ou seja,

$$
V=f+I .
$$

Da fórmula de Euler temos que $F=A-V+I$, aplicando as equações (1) e (2) obtemos a seguinte relação,

$$
\begin{aligned}
F & =\frac{1}{2}(3 F+f)-(f+I)+1 \\
& =\frac{3 F}{2}+\frac{f}{2}-f-I+1 .
\end{aligned}
$$

De onde segue

$$
F=f+2 I-2 .
$$

Como o número de faces $\mathrm{F}$ é igual ao número de triângulos fundamentais temos que a área do polígono é igual a 


$$
A(P)=\frac{1}{2} F,
$$

temos,

$$
A(P)=\frac{1}{2}(f+2 I-2),
$$

isto é,

$$
A(P)=\frac{f}{2}+I-1
$$

que é o Teorema de Pick.

\section{Uma aplicação do Teorema de Pick}

$\mathrm{Na}$ aritmética grande parte dos problemas são resolvidos através de equações do tipo

$$
a X-b Y=c \quad \text { ou } \quad a X+b Y=c,
$$

$\operatorname{com} a, b$ e $c$ pertencentes aos naturais.

Equações desse tipo são chamadas de equações diofantinas lineares. Mas nem sempre essas equações possuem soluções em números naturais. A proposição a seguir nos diz em que condições as equações do tipo

$$
a X-b Y=c
$$

possuem soluções inteiras.

Proposição 7.1 Sejam a e $b \in \mathbb{N}^{*} e c \in \mathbb{N}$. A equação $a X-b Y=c$ admite solução inteira se, e somente se, $\operatorname{MDC}(a, b) \mid c$.

Percebe-se que a equação $a X-b Y=c$, com $(a, b)=1$ admite infinitas soluções. Se $a, b$ e $c$ são números pequenos, a solução pode se determinada por tentativa, já que não se conhece uma fórmula simples para se encontrar tais soluções. Em geral é usado o algoritmo de Euclides, aqui proporemos o uso do teorema de Pick para determinar a solução minimal de uma equação diofantina.

Dada uma rede de pontos e um ponto $P(a, b)$ pertencente a rede, como $a$ e $b$ são primos entre si, vimos através da demonstração do Lema 3.1 que a reta $r$ que une o ponto $O(0,0)$ ao ponto $P$ não possui nenhum outro ponto da rede além dos pontos $P$ e $O$. Sendo assim movemos a reta $r$ paralelamente a si mesma, até encontrarmos o primeiro ponto $Q(t, s)$ pertencente a rede.
Percebemos então que os pontos $P, O$ e $Q$ são vértices de um triângulo primitivo, logo sua área é 1/2. Sabemos também que a área de um triângulo pode ser calculada através do cálculo do determinante de uma matriz formada pelas coordenadas dos vértices do triângulo, ou seja,

$$
A=\frac{|D|}{2}
$$

sendo assim, para

$$
D=\left(\begin{array}{lll}
0 & 0 & 1 \\
a & b & 1 \\
t & s & 1
\end{array}\right),
$$

temos $|D|=a s-b t$ e então

$$
A=\frac{a s-b t}{2}
$$

e como a área é igual a 1/2 temos,

$$
\frac{1}{2}=\frac{a s-b t}{2}
$$

o que implica

$$
a s-b t=1 \text {. }
$$

Comparando esse resultado com a equação

$$
a X+b Y=1,
$$

percebemos que os números $s$ e $t$ satisfazem essa equação, ou seja, $X=s$ e $Y=t$, note a inversão do par ordenado $(t, s)$.

Vejamos um exemplo que ilustra a utilização desse método.

Exemplo: Resolver a equação

$$
3 X-4 Y=10
$$

A equação tem solução, pois pela Proposição 7.1, $M D C(3,4) \mid 10$. Vamos encontrar a solução minimal desta equação. Pelo método exposto anteriormente temos: Primeiramente marcamos o ponto $P(3,4)$ numa rede, em seguida ligamos o ponto $O(0,0)$ ao ponto $P$, formando assim o segmento de reta $O P$, como na Figura 8. Sendo assim traçamos paralelamente a esse seguimento uma reta passando pelo ponto mais próximo de $O P$, notamos que esse ponto é (2,3), logo os números 2 e 3 satisfazem 


$$
3 a-4 b=1,
$$

então,

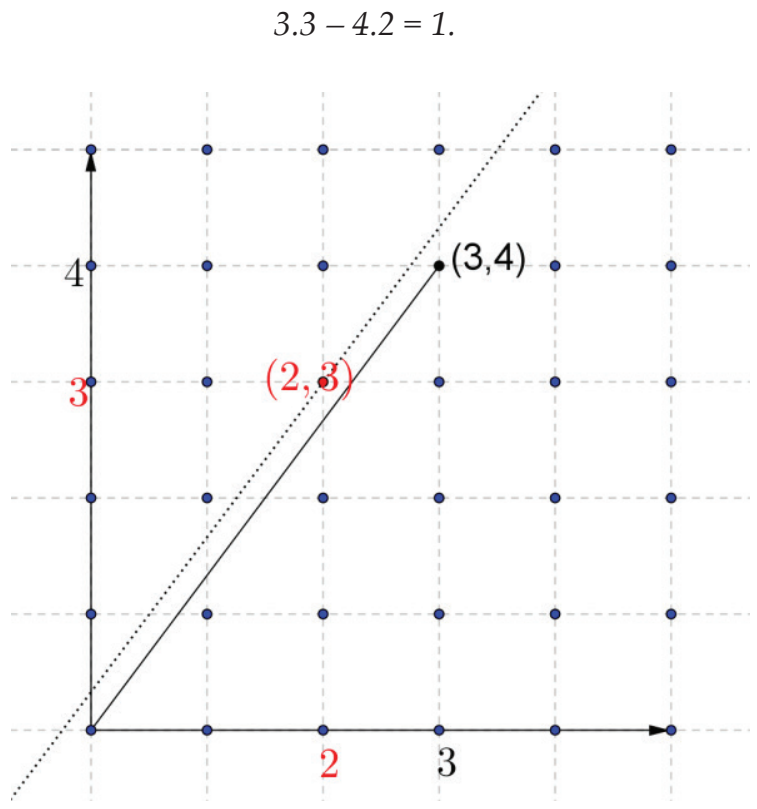

Figura 8

Multiplicando ambos os lados da equação

$$
3.3-4.2=1,
$$

por 10 temos

$$
3.30-4.20=10,
$$

$\operatorname{logo} x_{1}=30$ e $x_{2}=20$ é solução particular da equação. Portanto, as soluções $x, y$ em $N$ da equação são $x=30-4 t$ e $y=20-3 t$, com $t$ pertencente aos $N$. Para encontrarmos a solução minimal determinaremos o maior valor de $t$, de modo $x, y$ pertençam aos $N$. Isto ocorre quando $t=6, \log$ a solução minimal é $x_{0}=6$ e $y_{0}=2$ e as soluções da equação são, $x=6+4 t$ e $y=2+3 t$.

\section{Conclusões}

Pela observação dos tópicos abordados neste trabalho, entende-se que o Teorema de Pick pode ser explorado de várias formas e níveis de profundidade. Pois percebemos que ao mesmo tempo em que o teorema apresenta uma série de relações com outros conteúdos da Matemática a sua simplicidade também permite que o tema seja trabalhado de forma lúdica, até mesmo nas séries iniciais. Vimos ainda que a tentativa de demonstra-lo nos levou a formular ideias e argumentos de forma clara e precisa, o que é um dos objetivos no ensino da Matemática.

\section{Apêndice}

Ao decompormos o polígono em triângulos fundamentais, vimos anteriormente na demonstração do teorema de Pick que o número de triângulo no qual no qual o polígono pode ser decomposto é igual a $f+2 I-2$, onde $f$ é o número de pontos da fronteira e $I$ o número de pontos do interior do polígono. Mas esse número de triângulos é igual ao número de faces do poliedro plano, ou seja,

$$
F=f+2 I-2
$$

Temos ainda que o número de vértices do poliedro é igual ao número de pontos da fronteira mais o número de pontos no interior,

$$
V=I+f
$$

Analisando o número de arestas, percebemos que cada aresta externa $A_{e}$ é lado de apenas uma face e que cada aresta interna $A_{i}$ é lado de duas faces, com isso temos a seguinte relação,

$$
3 F=A_{e}+2 A_{i}
$$

Mas o número de arestas internas $A_{i}$ é igual ao número total de arestas $A$ menos o número de arestas externas $A_{e}$

$$
3 F=A_{e}+2\left(A-A_{e}\right),
$$

Além disso, o número de arestas externas $A_{e}$ é igual ao número de pontos na fronteira $f$, então,

$$
\begin{aligned}
& 3 F=f+2(A-f), \\
& 3 F=f+2 A-2 f, \\
& 3 F=2 A-f .
\end{aligned}
$$

Aplicando a relação (3) temos,

$$
\begin{aligned}
3(f+2 I-2) & =2 A-f \\
3 f+6 I-6 & =2 A-f \\
2 A & =4 f+6 I-6 .
\end{aligned}
$$


Logo temos

$$
A=2 f+3 I-3
$$

Logo, de (3), (4), (5) segue que

$V+F-A=I+f+f+2 I-2(2 f+3 I-3)$

$V+F-A=3 I+2 f-2-2 f-3 I+3$

$V+F-A=1$.

\section{Referências}

[1] E. L. Lima; Meu Professor de Matemática e outras histórias, IMPA e Fundação Vitae, 1991.

[2] A. L. Pereira e S. T. Melo; Contando Áreas - O Teorema de Pick. Revista do Professor de Matemática, São Paulo, n. 78, p.36-42, 2o quadrimestre. 2012.

[3] T. M. Rocha e Andrade; Áreas das noções intuitivas ao Teorema de Pick. Acesso em 07 dezembro de 2013. $<$ wwww.diaadiaeducação.pr.gov.br/portals/pde/arqu ivos/1mad23-4.pdf>

[4] J. N. Tavares; Teorema de Pick. Acesso em 05 novembro de 2013. $<$ http://cmup.fc.up.pt/cmup/pick/index.html>.

[5] D. C. O. Leite; Teorema de Pick: Relato de uma Pratica Investigativa.

[6] LEITE, Danielle Cristina Oliveira. Teorema de Pick: Relato de uma Prática investigativa. 